\title{
Mathematical model of a drive mechanism with a crank device of a crank press
}

\author{
Kirill Kobzev ${ }^{1, *}$ \\ ${ }^{1}$ Don State Technical University, 344002, Gagarina Sq., 1, Rostov-on-Don, Russia
}

\begin{abstract}
The most important link in the forging equipment is a crank mechanism. Their significant drawback is the unbalanced inertia forces of the moving masses of the crank mechanism, which cause vibration. The analysis of the phenomena occurring in the mechanism and the assessment of the technological process are based on the theory of chains, which allows analytically analyzing the dynamic characteristics of systems with a large number of degrees of freedom, based on the analysis of one structural element. The study of the process of force interaction inevitably comes down to the construction of a mathematical model of mechanisms, the formative movement of which leads to its formation. One of the partial systems makes an irregular programmed motion, meaning the crank drive mechanism. In addition, unwanted vibrations caused by kinematic excitation are superimposed on this drive. According to numerous papers on this topic, significant dynamic errors arise due to vibration accelerations. One of the main tasks in reducing the vibration activity and, accordingly, the level of acoustic emission of the process under study is to ensure the required law of motion of the instrument. On this basis, the study of the stability of formative movements is of particular importance. This question is complicated by the fact that in the processing, there is a change in the process parameters and, consequently, in the characteristics of the friction coupling. The latter circumstance presupposes the evolution of the system under study, and therefore the need for process control.
\end{abstract}

\section{Introduction}

The most important link in the forging equipment is a crank mechanism. Their significant drawback is the unbalanced inertia forces of the moving masses of the crank mechanism, which cause vibration. The analysis of the phenomena occurring in the mechanism and the assessment of the technological process are based on the theory of chains, which allows analytical-ly analyzing the dynamic characteristics of systems with a large number of degrees of freedom, based on the analysis of one structural element.

The study of the process of force interaction inevitably comes down to the construction of a mathematical model of mechanisms, the formative movement of which leads to its formation. One of the partial systems makes an irregular programmed motion, meaning the crank drive mechanism. In addition, unwanted vibrations caused by kinematic excitation

\footnotetext{
*Corresponding author: 5976765@mail.ru
} 
are superimposed on this drive. According to numerous papers on this topic, especially significant dynamic errors arise due to vibration accelerations.

One of the main tasks in reducing the vibration activity, and, accordingly, the level of acoustic emission, of the process under study is to ensure the required law of motion of the instrument. On this basis, of particular importance is the study of the stability of formative movements. This question is further complicated by the fact that in the process of processing there is a change in the process parameters and, consequently, in the characteristics of the friction coupling. The latter circumstance presupposes the evolution of the system under study, and therefore the need for process control.

In the study of the dynamics of the elastic elements of machines and mechanisms, as a rule, it is assumed that the influence of the devices forcing the oscillations is unilateral, that is, there is no reverse effect of the elastic subsystem on the energy source.

Systems that have a source of energy, the inverse effect on which from the other links of the mechanical system cannot be neglected due to its final power, are called systems with limited excitation (or non-ideal systems). As practice shows, in some cases, a sharp increase in the amplitudes of oscillations of elastic elements of a non-ideal system, which arose when the operating mode enters the resonance region, can lead to dangerous modes of oscillation.

\section{Materials and methods}

Consider the drive mechanism, which is a serially connected belt drive, and a crank mechanism, which implements the kinematic function of the position of the driven link.

The considered mechanism belongs to the class of devices that convert the rotational movement of the drive shaft into an uneven movement of the working body. In this case, the dependence of the form $V=\Pi(\varphi)$, connecting the position of the slave link with the angle of rotation of the drive motor is nonlinear. It is usually called the position function, and its angle derivatives $\varphi$ respectively $\frac{d \Pi(\varphi)}{d \varphi}$ и $\frac{d_{2} \Pi(\varphi)}{d \varphi^{2}}$ called transfer functions. Analysis of the kinematic scheme of the mechanism under study allows us to conclude that the dynamic can be classified as a nonholonomic system, due to the presence of a belt transmission, with scleronomic connections. However, it is necessary to take into account the possibility of manifestation of parametric resonances, since the reduced moment of inertia of the mechanism is also a function $\varphi$. It can also be classified as a vibratory machine with kinematic excitation. The interaction of the working body with the material creates a technological load on the oscillatory system. In this case, the dynamic model of the object under study can be represented as figure 1 : 


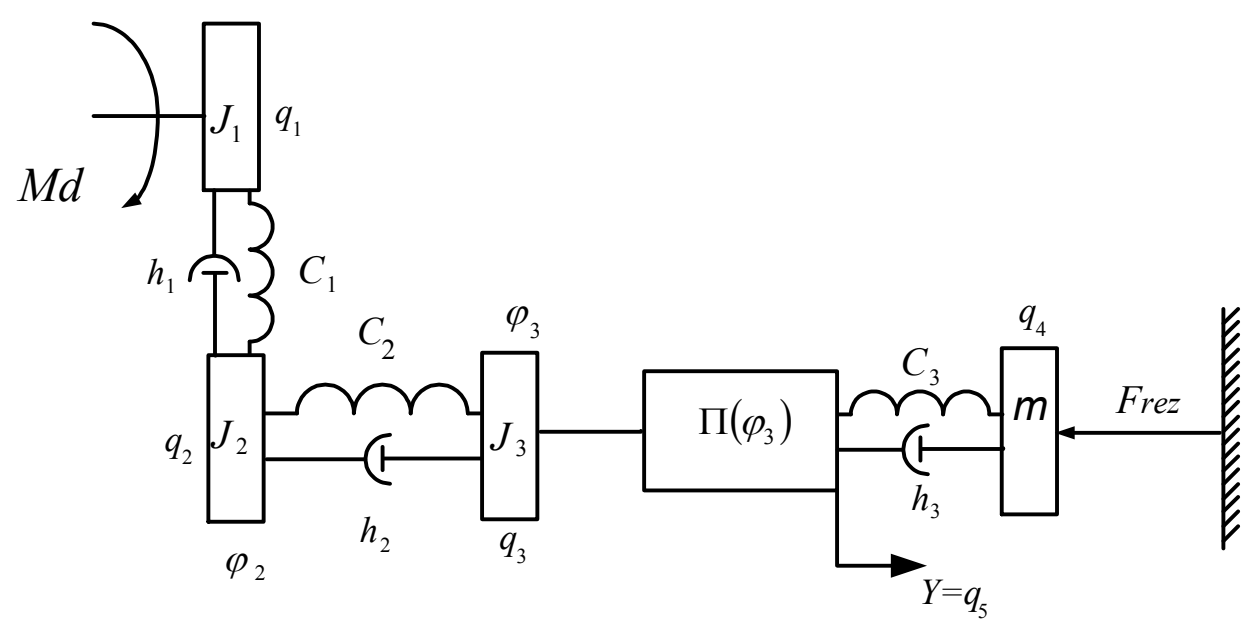

Fig. 1. The design scheme of the drive.

Since the links of this mechanism perform complex flat movement in a vertical plane, it is necessary to take into account the work of gravity forces. In addition, the mechanism has a link that performs a plane-parallel motion. For such links, their inertial characteristics can be reduced to links that perform rotational and translational motion, that is, to a crank and frame. Using the mass substitution method, we get:

$$
\Delta J_{3}=m_{u} \cdot \frac{L}{2} \cdot R_{3}^{2} \quad \Delta m=m_{u} \cdot \frac{L}{2} .
$$

The electromechanical system cannot be described by an adequate model without taking into account the inertia of the processes occurring in the engine. The equations describing the motion of an asynchronous electric motor have the form:

$$
\begin{gathered}
\frac{d \Psi_{1 x}}{d t}=U_{1 x}-\frac{r_{1} \cdot L_{2}^{\prime} \cdot \Psi_{1 x}}{\Delta}+\frac{r_{1} \cdot L_{0} \cdot \Psi_{2 x}}{\Delta}+\Omega_{0} \cdot \Psi_{1 y} \\
\frac{d \Psi_{1 y}}{d t}=U_{1 y}-\frac{r_{1} \cdot L_{2}^{\prime} \cdot \Psi_{1 y}}{\Delta}+\frac{r_{1} \cdot L_{0} \cdot \Psi_{2 y}}{\Delta}-\Omega_{0} \cdot \Psi_{1 x}, \\
\frac{d \Psi_{2 x}}{d t}=-\frac{r_{2}^{\prime} \cdot L_{1} \cdot \Psi_{2 x}}{\Delta}+\frac{r_{2}^{\prime} \cdot L_{0} \cdot \Psi_{1 x}}{\Delta}+\left(\Omega_{0}-\Omega\right) \cdot \Psi_{2 y}, \\
\frac{d \Psi_{2 y}}{d t}=-\frac{r_{2}^{\prime} \cdot L_{1} \cdot \Psi_{2 y}}{\Delta}+\frac{r_{2}^{\prime} \cdot L_{0} \cdot \Psi_{1 y}}{\Delta}-\left(\Omega_{0}-\Omega\right) \cdot \Psi_{2 x}, \\
U_{1 x}=\sqrt{2} \cdot U_{m} \cdot \cos \omega_{p} \cdot t \quad U_{1 y}=\sqrt{2} \cdot U_{m} \cdot \sin \omega_{p} \cdot t \\
\frac{d \omega}{d t}=\frac{3 \cdot Z_{p}}{2} \cdot \frac{L_{0}}{J \cdot \Delta} \cdot\left(\Psi_{2 x} \cdot i_{1 y}-\Psi_{2 y} \cdot i_{1 x}\right)-\frac{1}{J} \cdot M_{C},
\end{gathered}
$$


where

$-Z_{p}$ - the number of pole pairs of the electric motor;

- $\Psi_{1 x}$ - the projection of the flux linkage of the stator winding on the axis $x$;

- $\Psi_{1 y}$ - the projection of the flux linkage of the stator winding on the axis $y$;

- $\Psi_{2 x}$ - the projection of the flux linkage of the stator winding on the axis $x$;

- $\Psi_{2 y}$ - the projection of the flux linkage of the stator winding on the axis $y$;

- $J$ - the sum of the moment of inertia of the rotor of the electric motor and the pulley;

$-\Omega_{0}$ - angular frequency of rotation of the stator field;

- $\Omega$ - angular frequency of rotation of the rotor;

$-\omega_{p}$ - angular frequency.

Thus, the electromechanical system under investigation includes an electric motor described by a system of nonlinear differential equations of the first order, as well as a system of equations describing the mechanical part of the drive. For such problems, a special form of the Lagrange type II equations with "superfluous" coordinates is usually used (they are also called Ferrers equations). For the case in question, we have:

$$
\begin{aligned}
& \frac{d}{d t}\left(\frac{\partial T}{\partial \dot{q}_{k}}\right)-\frac{\partial T}{\partial q_{k}}+\frac{\partial P}{\partial q_{k}}+\frac{\partial \Phi}{\partial \dot{q}_{k}}=Q_{k}+\sum_{j=1}^{n} \lambda_{j} a_{j k} \quad(k=1, \ldots, s) \\
& \sum_{k=1}^{s} a_{j k} \dot{q}_{k}+a_{j}=0 \quad(j=1, \ldots, n)
\end{aligned}
$$

We specify the absolute coordinate corresponding to the displacement at the beginning of the kinematic chain, and then enter the coordinates, moving along the kinematic chain. We write the basic kinematic relations:

$$
\begin{gathered}
\varphi_{1}=\Omega \cdot t=q_{1}, \\
\varphi_{2}=\varphi_{1} i_{1}+q_{2}=q_{1} i_{1}+q_{2}, \\
\varphi_{3}=\varphi_{1} \cdot i_{1}+q_{2}+q_{3}=q_{1} i_{1}+q_{2}+q_{3}, \\
Y=\Pi\left(\varphi_{3}\right)=\Pi\left(q_{1} i_{1}+q_{2}+q_{3}\right)=q_{5}, \\
Z=Y+q_{4} \quad Z=q_{5}+q_{4} .
\end{gathered}
$$

The angular coordinates of the corresponding sections in absolute motion, - the absolute coordinate of the mass. The last remark means that, with the exception of the generalized coordinates, relative coordinates are taken which are responsible for the deformation of the elastic elements. 
The equation of connection is written based on the condition that is a function of the position.

$$
\Pi\left(q_{1} i_{1}+q_{2}+q_{3}\right)-Z=\Pi\left(\varphi_{3}\right)-Z=0 .
$$

We differentiate this expression by time:

$$
\Pi^{\prime} \dot{q}_{1} i_{1}+\Pi^{\prime} \dot{q}_{2}+\Pi^{\prime} \dot{q}_{3}-\dot{Z}=0 .
$$

As the "extra" coordinates, we take $Y=q_{5}$. The number of degrees of freedom of the system under study $H=4$, the number of "extra" coordinates $n=1$.

Due to the fact that the frame with moves in the vertical plane of Fig.1.2, it is convenient to present its movement in the following form:

$$
S=L \cdot \cos \delta+R \cdot \sin \varphi
$$
form:

The common leg of the $\mathrm{OAC}$ and $\mathrm{ABC}$ rectangles is determined by the equality of the

$$
\begin{aligned}
& \sin \delta=\varsigma \cdot \cos \varphi, \quad \text { where: } \\
& \varsigma=\frac{R}{L}, \delta=\arcsin (\varsigma \cdot \cos \varphi) .
\end{aligned}
$$

The transfer function of the drive mechanism can be represented as a function of the angle of rotation of the crank:

$$
\begin{array}{r}
\Pi(\varphi)=S=L \cdot \cos \delta+R \cdot \sin \varphi-\sqrt{L^{2}-R^{2}} \\
\Pi(\varphi)=L \cdot \sqrt{1-\varsigma^{2} \cdot \cos ^{2} \varphi}+R \cdot \sin \varphi-\sqrt{L^{2}-R^{2}}
\end{array}
$$

Making obvious transformations, we have:

$$
\begin{gathered}
\frac{d S}{d \varphi}=-L \sin \delta \cdot \frac{d \delta}{d \varphi}+R \cos \varphi \\
\frac{d^{2} S}{d \varphi^{2}}=-L \sin \delta \cdot \frac{d^{2} \delta}{d \varphi^{2}}-L \cos \delta \cdot\left(\frac{d \delta}{d \varphi}\right)^{2}-R \sin \varphi \\
\frac{d}{d \varphi}(\sin \delta)=\frac{d}{d \varphi}(\varsigma \cdot \cos \varphi)=-\varsigma \cdot \sin \varphi \\
\frac{d \delta}{d \varphi} \cos \delta=-\varsigma \cdot \sin \varphi \quad \text { or } \frac{d \delta}{d \varphi}=-\varsigma \cdot \frac{\sin \varphi}{\cos \delta}
\end{gathered}
$$




$$
\Pi^{\prime}(\varphi)=-L \cdot \frac{d \delta}{d \varphi} \sin \delta+R \cdot \cos \varphi=L \cdot \varsigma \cdot \frac{\sin \varphi}{\cos \delta} \cdot \sin \delta+R \cdot \cos \varphi
$$

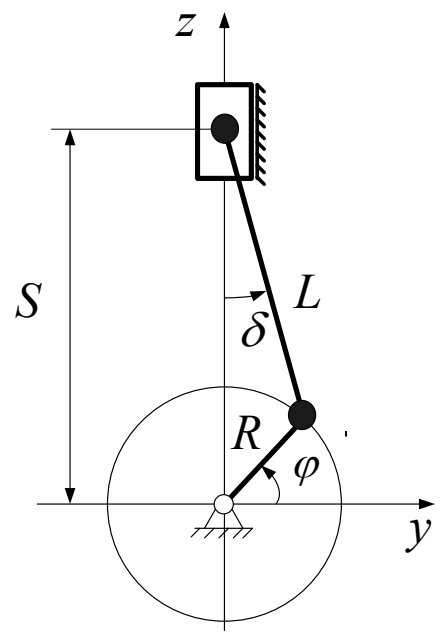

Fig. 2. Crank mechanism.

For the second derivative of the transfer function, respectively, we obtain:

$$
\begin{gathered}
\frac{d}{d \varphi}\left(\frac{d \delta}{d \varphi} \cdot \cos \delta\right)=\frac{d}{d \varphi}(-\varsigma \cdot \sin \varphi) \\
\frac{d^{2} \delta}{d \varphi^{2}} \cdot \cos \delta-\left(\frac{d \delta}{d \varphi}\right)^{2} \cdot \sin \delta=-\varsigma \cdot \cos \varphi \\
\Pi^{\prime \prime}(\varphi)=\frac{d^{2} S}{d \varphi^{2}}=-L \cdot \sin \delta \cdot \frac{d^{2} \delta}{d \varphi^{2}}=\frac{1}{\cos \delta} \cdot\left(\varsigma^{2} \cdot\left(\frac{\sin \varphi}{\cos \delta}\right)^{2} \cdot \sin \delta-\varsigma \cdot \cos \varphi\right) \\
\Pi^{\prime \prime}(\varphi)=-L \cdot \frac{\sin \delta}{\cos \delta} \cdot\left(\varsigma^{2} \cdot\left(\frac{\sin \varphi}{\cos \delta}\right)^{2} \cdot \sin \delta-\varsigma \cdot \cos \varphi\left(-L \cos \delta \cdot \varsigma^{2} \cdot\left(\frac{\sin \varphi}{\cos \delta}\right)^{2}-R \cdot \sin \varphi\right.\right.
\end{gathered}
$$

To compile a mathematical model of the mechanical part of the drive, we express the kinetic and potential energy in terms of the accepted generalized coordinates, including the "redundant" ones:

$$
2 T=J_{1} \dot{\varphi}_{1}^{2}+J_{2} \dot{\varphi}_{2}^{2}+J_{3} \dot{\varphi}_{3}^{2}+m \cdot \dot{Z}^{2}
$$




$$
2 P=C_{1}\left(\varphi_{2}-\varphi_{1}\right)^{2}+C_{2}\left(\varphi_{3}-\varphi_{2}\right)^{2}+C_{3} \cdot(Z-Y)^{2}
$$

System dissipative function (Rayleigh function):

$$
2 \Phi=h_{1}\left(\dot{\varphi}_{2}-\dot{\varphi}_{1}\right)^{2}+h_{2}\left(\dot{\varphi}_{3}-\dot{\varphi}_{2}\right)^{2}+h_{3}(\dot{Z}-\dot{Y})^{2} .
$$

The expression for virtual work can be written in the form:

$$
\delta A=M d \cdot \delta q_{1}-F_{p e z} \cdot \delta Z-b_{2} \cdot \dot{q}_{2} \cdot \delta q_{2}-b_{4} \dot{Z} \cdot \delta Z
$$

We write the expression of kinetic energy, taking into account the kinematic relations:

$$
\begin{aligned}
& \quad 2 T=J_{1} \dot{q}_{1}^{2}+J_{2}\left(\dot{q}_{1} i_{1}+\dot{q}_{2}\right)^{2}+J_{3}\left(\dot{q}_{1} i_{1}+\dot{q}_{2}+\dot{q}_{3}\right)^{2}+m \cdot\left(\dot{q}_{4}+\dot{q}_{5}\right)^{2}, \\
& 2 T=J_{1} \dot{q}_{1}^{2}+J_{2}\left(\dot{q}_{1}^{2} \dot{i}_{1}^{2}+2 i_{1} \dot{q}_{1} \dot{q}_{2}+\dot{q}_{2}^{2}\right)+J_{3}\left(\dot{q}_{1}^{2} \dot{i}_{1}^{2}+2 i_{1} \dot{q}_{1} \cdot\left(\dot{q}_{2}+\dot{q}_{3}\right)+\dot{q}_{2}^{2}+\dot{q}_{3}^{2}+2 \dot{q}_{2} \dot{q}_{3}\right)+ \\
& +m \cdot\left(\dot{q}_{5}^{2}+2 \dot{q}_{4} \dot{q}_{5}+q_{4}^{2}\right) \\
& 2 T=\dot{q}_{1}^{2}\left(J_{1}+J_{2} i_{1}^{2}+J_{3} \dot{i}_{1}^{2}\right)+\dot{q}_{2}^{2}\left(J_{2}+J_{3}\right)+\dot{q}_{3}^{2} J_{3}+2 \dot{q}_{1} \dot{q}_{2}\left(J_{2} i_{1}+J_{3} i_{1}\right)+2 \dot{q}_{2} \dot{q}_{3} J_{3}+ \\
& +2 \dot{q}_{1} \dot{q}_{3} i_{1} J_{3}+m \dot{q}_{5}^{2}+2 m \dot{q}_{4} \dot{q}_{5}+m \dot{q}_{4}^{2}
\end{aligned}
$$

Based on the matrix representation of the kinetic energy of the system $2 T=\dot{\mathbf{q}}^{T} \cdot \mathbf{M n} \cdot \dot{\mathbf{q}}$ and the fact that the coefficients at generalized speeds are constant, the inertial coefficients equating the corresponding coefficients of the quadratic form representing the kinetic energy of the system, we obtain the matrix of inertial coefficients in the form:

$$
\mathbf{M n}=\left[\begin{array}{cccc}
J_{1}+J_{2} i_{1}^{2}+J_{3} i_{1}^{2} & J_{2} i_{1}+J_{3} i_{1} & J_{3} i_{1} & 0 \\
J_{2} i_{1}+J_{3} i_{1} & J_{2}+J_{3} & J_{3} & 0 \\
J_{3} i_{1} & J_{3} & J_{3} & 0 \\
0 & 0 & 0 & m
\end{array}\right]
$$

It is necessary to consider the counting of potential energy from the position of stable equilibrium. In addition, we believe that all communications are stationary, the process is not considered as a component of the mechanism, but as an external influence. If we assume that the gear ratio does not depend on the speed of the links, the stiffness matrix of the approximating system of differential equations of the object under study can be taken as constant.

$$
\begin{gathered}
2 P=C_{1}\left(\varphi_{2}-\varphi_{1}\right)^{2}+C_{2}\left(\varphi_{3}-\varphi_{2}\right)^{2}+C_{3}(Z-Y)^{2}, \\
2 P=C_{1}\left(q_{1}\left(i_{1}-1\right)+q_{2}\right)^{2}+C_{2}\left(q_{1} i_{1}+q_{2}+q_{3}-q_{1} i_{1} q_{2}\right)^{2}+C_{3}\left(q_{5}+q_{4}-q_{5}\right)^{2},
\end{gathered}
$$




$$
2 P=q_{1}^{2} \cdot C_{1} \cdot\left(i_{1}-1\right)^{2}+q_{1} q_{2} C_{1}\left(i_{1}-1\right)+q_{2}^{2} \cdot C_{1}+q_{3}^{2} \cdot C_{2}+q_{4}^{2} \cdot C_{3} .
$$

Based on the matrix representation of the potential energy of the system and the fact that the stiffnesses of the bonds are constant, the coefficients of the stiffness matrix are obtained by equating the corresponding coefficients of the quadratic form representing the potential energy of the system, in the form:

$$
\mathbf{C}=\left[\begin{array}{cccc}
C_{1} \cdot\left(i_{1}-1\right)^{2} & C_{1} \cdot\left(i_{1}-1\right) & 0 & 0 \\
C_{1} \cdot\left(i_{1}-1\right) & C_{1} & 0 & 0 \\
0 & 0 & C_{2} & 0 \\
0 & 0 & 0 & C_{3}
\end{array}\right]
$$

The coordinate entered into the expression of potential energy, but although it is rigidly connected with the coordinate by a nonlinear function, the change in the potential energy of the system under study is determined by the movement of inertial masses in the vertical plane.

Imagine the damping function in the matrix form:

$$
\begin{gathered}
2 \Phi=h_{1}\left(\dot{\varphi}_{2}-\dot{\varphi}_{1}\right)^{2}+h_{2}\left(\dot{\varphi}_{3}-\dot{\varphi}_{2}\right)^{2}+h_{3}(\dot{Z}-\dot{Y})^{2}, \\
2 \Phi=h_{1}\left(\dot{q}_{1}\left(i_{1}-1\right)+\dot{q}_{2}\right)^{2}+h_{2}\left(\dot{q}_{1} i_{1}+\dot{q}_{2}+\dot{q}_{3}-\dot{q}_{1} i_{1} \dot{q}_{2}\right)^{2}+h_{3}\left(\dot{q}_{5}+\dot{q}_{4}-\dot{q}_{5}\right)^{2} \\
2 \Phi=\dot{q}_{1}^{2} \cdot h_{1} \cdot\left(i_{1}-1\right)^{2}+\dot{q}_{2}^{2} \cdot h_{1}+\dot{q}_{3}^{2} \cdot h_{2}+h_{3} \cdot \dot{q}_{3}^{2}+2 \dot{q}_{1} \dot{q}_{2} \cdot h_{1}\left(i_{1}-1\right) .
\end{gathered}
$$

Based on the representation of the damping matrix in the form, we obtain the matrix in the form:

$$
\mathbf{H n}=\left[\begin{array}{cccc}
h_{1} \cdot\left(i_{1}-1\right)^{2} & h_{1} \cdot\left(i_{1}-1\right) & 0 & 0 \\
h_{1} \cdot\left(i_{1}-1\right) & h_{1} & 0 & 0 \\
0 & 0 & h_{2} & 0 \\
0 & 0 & 0 & h_{3}
\end{array}\right]
$$

The moments of friction forces in the supports neglected. Further actions are reduced to writing a system of differential equations of motion and the elimination of Lagrange multipliers. The total number of equations is. In the first part, in addition to the generalized forces, there is a summand. Let us write the equations that establish the connection between "extra" and independent coordinates.

Determine the coefficients of the equations of additional relationships:

$$
\begin{gathered}
Y=q_{5}=\Pi(\varphi)=\Pi\left(q_{1} i_{1}+q_{2}+q_{3}\right), \\
\dot{q}_{5}=\Pi^{\prime}\left(\varphi_{3}\right) \cdot \dot{q}_{1} i_{1}+\Pi^{\prime}\left(\varphi_{3}\right) \cdot \dot{q}_{2}+\Pi^{\prime}\left(\varphi_{3}\right) \cdot \dot{q}_{3} \text { or } \\
\Pi^{\prime}\left(\varphi_{3}\right) \cdot \dot{q}_{1} i_{1}+\Pi^{\prime}\left(\varphi_{3}\right) \cdot \dot{q}_{2}+\Pi^{\prime}\left(\varphi_{3}\right) \cdot \dot{q}_{3}-\dot{q}_{5}=0 .
\end{gathered}
$$


For further discussion, it is convenient to present this equality in the matrix form:

$$
\begin{gathered}
\Pi^{\prime}\left(\varphi_{3}\right) \cdot\left[\begin{array}{llll}
i_{1} & 1 & 1 & 0
\end{array}\right] \cdot\left[\begin{array}{c}
\dot{q}_{1} \\
\dot{q}_{2} \\
\dot{q}_{3} \\
\dot{q}_{4}
\end{array}\right]-\dot{q}_{5}=\Pi^{\prime}\left(\varphi_{3}\right) \cdot \mathbf{I s} \cdot \dot{\mathbf{q}}-\dot{q}_{5}=0 \\
\dot{q}_{5}=\Pi^{\prime}\left(\varphi_{3}\right) \cdot \mathbf{I s} \cdot \dot{\mathbf{q}} \\
\ddot{q}_{5}=\Pi^{\prime \prime}\left(\varphi_{3}\right) \cdot \mathbf{I s} \cdot \dot{\mathbf{q}}+\Pi^{\prime}\left(\varphi_{3}\right) \cdot \mathbf{I s} \cdot \ddot{\mathbf{q}}
\end{gathered}
$$

General view of the equation of communication:

$$
\sum_{k=1}^{H+n} a_{k} \dot{a}_{k}+a 1=0, \text { where: } H+n=5 .
$$

Let us compare the result obtained with the equation of connection in general form. Given that the index of the coefficient corresponds to the generalized speed, we write:

$$
a_{1} \dot{q}_{1}+a_{2} \dot{q}_{2}+a_{3} \dot{q}_{3}+a_{4} \dot{q}_{4}+a_{5} \dot{q}_{5}+a 1=0
$$

From the last equality follows:

$$
\begin{gathered}
a_{1}=\Pi^{\prime}\left(\varphi_{3}\right) \cdot i_{1} \quad a_{2}=\Pi^{\prime}\left(\varphi_{3}\right) \quad a_{3}=\Pi^{\prime}\left(\varphi_{3}\right), \\
a_{4}=0 \quad a_{5}=-1 .
\end{gathered}
$$

The definition of generalized forces is carried out, making the expression of the amount of work on virtual displacements:

$$
\begin{aligned}
& \delta A=M d \cdot \delta q_{1}-m g \cdot \delta\left(q_{4}+q_{5}\right)-\text { Frez } \cdot \delta\left(q_{4}+q_{5}\right) \\
& Q_{1}=M d \quad Q_{4}=-m g-\text { Frez } Q_{5}=-m g-\text { Frez }
\end{aligned}
$$

Finally, the system of equations of the forced motion of the mechanism can be written in a matrix form, highlighting the fifth equation, which is intended to determine the Lagrange multiplier:

$$
\begin{gathered}
\mathbf{M n} \cdot \ddot{\mathbf{q}}+\mathbf{H n} \cdot \dot{\mathbf{q}}+\mathbf{C q}=\left[\begin{array}{c}
M d+\Lambda a_{1} \\
\Lambda a_{2} \\
\Lambda a_{3} \\
-m g-F r e z+\Lambda a_{4}
\end{array}\right]=\left[\begin{array}{c}
M d+\Lambda \Pi^{\prime} i_{1} \\
\Lambda \Pi^{\prime} \\
\Lambda \Pi^{\prime} \\
-m g-F r e z
\end{array}\right], \\
m \ddot{q}_{4}+m \ddot{q}_{5}=-m g-F r e z-\Lambda
\end{gathered}
$$


The last equation of the reduced system is used to determine the Lagrange multiplier:

$$
\Lambda=-m \ddot{q}_{4}-m \ddot{q}_{5}-m g-\text { Frez }
$$

Finally, the system of equations of the power-saw bench drive is obtained in the form of matrix equality:

$$
\left(\mathbf{M n} \cdot p^{2}+\mathbf{H n} \cdot p+\mathbf{C}\right) \cdot \mathbf{q}=\mathbf{F}(\mathbf{X}, t) .
$$

Write the expression for the given value of the Lagrange multiplier:

$$
\begin{gathered}
\mathbf{F}(\mathbf{q}, t)=\left[\begin{array}{c}
M d+\Lambda \Pi^{\prime} i_{12} \\
\Lambda \Pi^{\prime} \\
\Lambda \Pi^{\prime} \\
-m g-F r e z
\end{array}\right]=\left[\begin{array}{c}
M d-\Pi^{\prime} i_{12} \cdot\left(m \ddot{q}_{4}+m \ddot{q}_{5}+m g+F r e z\right) \\
-\Pi^{\prime} \cdot\left(m \ddot{q}_{4}+m \ddot{q}_{5}+m g+F r e z\right) \\
-\Pi^{\prime} \cdot\left(m \ddot{q}_{4}+m \ddot{q}_{5}+m g+F r e z\right) \\
-m g-F r e z
\end{array}\right]= \\
=\left[\begin{array}{c}
M d-\Pi^{\prime} \cdot i_{12} \cdot(m g+F r e z) \\
-\Pi^{\prime} \cdot(m g+F r e z) \\
-\Pi^{\prime} \cdot(m g+F r e z) \\
-m g-F r e z
\end{array}\right]-m \Pi^{\prime} \cdot\left[\begin{array}{c}
i_{12} \cdot\left(\ddot{q}_{4}+\ddot{q}_{5}\right) \\
\ddot{q}_{4}+\ddot{q}_{5} \\
\ddot{q}_{4}+\ddot{q}_{5} \\
0
\end{array}\right]
\end{gathered}
$$

We will transform the vector defined by the "extra" coordinate, provided that

$$
\begin{aligned}
& \ddot{q}_{5}=\Pi^{\prime \prime}\left(\varphi_{3}\right) \cdot \mathbf{I s} \cdot \dot{\mathbf{q}}+\Pi^{\prime}\left(\varphi_{3}\right) \cdot \mathbf{I s} \cdot \ddot{\mathbf{q}}: \\
& m \Pi^{\prime} \cdot\left[\begin{array}{c}
i_{12} \cdot\left(\ddot{q}_{4}+\ddot{q}_{5}\right) \\
\ddot{q}_{4}+\ddot{q}_{5} \\
\ddot{q}_{4}+\ddot{q}_{5} \\
0
\end{array}\right]=m \Pi^{\prime} \cdot\left[\begin{array}{c}
\dot{1}_{12} \ddot{q}_{4}+i_{12} \cdot \Pi^{\prime}\left(\ddot{q}_{1} \cdot i_{12}+\ddot{q}_{2}+\ddot{q}_{3}\right)+i_{12} \cdot \Pi^{\prime \prime}\left(\dot{q}_{1} \cdot \dot{i}_{12}+\dot{q}_{2}+\dot{q}_{3}\right) \\
\ddot{q}_{4}+i_{12} \cdot \Pi^{\prime}\left(\ddot{q}_{1} \cdot \dot{1}_{12}+\ddot{q}_{2}+\ddot{q}_{3}\right)+\Pi^{\prime \prime}\left(\dot{q}_{1} \cdot i_{12}+\dot{q}_{2}+\dot{q}_{3}\right) \\
\ddot{q}_{4}+i_{12} \cdot \Pi^{\prime}\left(\ddot{q}_{1} \cdot \dot{i}_{12}+\ddot{q}_{2}+\ddot{q}_{3}\right)+\Pi^{\prime \prime}\left(\dot{q}_{1} \cdot i_{12}+\dot{q}_{2}+\dot{q}_{3}\right) \\
0
\end{array}\right]= \\
& =m \Pi^{\prime} \cdot\left[\begin{array}{cccc}
i_{12}{ }^{2} \cdot \Pi^{\prime} & i_{12} \cdot \Pi^{\prime} & i_{12} \cdot \Pi^{\prime} & i_{12} \\
i_{12}{ }^{2} \cdot \Pi^{\prime} & i_{12} \cdot \Pi^{\prime} & i_{12} \cdot \Pi^{\prime} & 1 \\
i_{12}{ }^{2} \cdot \Pi^{\prime} & i_{12} \cdot \Pi^{\prime} & i_{12} \cdot \Pi^{\prime} & 1 \\
0 & 0 & 0 & 0
\end{array}\right] \cdot\left[\begin{array}{l}
\ddot{q}_{1} \\
\ddot{q}_{2} \\
\ddot{q}_{3} \\
\ddot{q}_{4}
\end{array}\right]+m \Pi^{\prime} \cdot\left[\begin{array}{cccc}
i_{12}{ }^{2} \cdot \Pi^{\prime \prime} & i_{12} \cdot \Pi^{\prime \prime} & i_{12} \cdot \Pi^{\prime \prime} & 0 \\
i_{12}{ }^{2} \cdot \Pi^{\prime \prime} & i_{12} \cdot \Pi^{\prime \prime} & i_{12} \cdot \Pi^{\prime \prime} & 0 \\
i_{12}{ }^{2} \cdot \Pi^{\prime \prime} & i_{12} \cdot \Pi^{\prime \prime} & i_{12} \cdot \Pi^{\prime \prime} & 0 \\
0 & 0 & 0 & 0
\end{array}\right] \cdot\left[\begin{array}{c}
\dot{q}_{1} \\
\dot{q}_{2} \\
\dot{q}_{3} \\
\dot{q}_{4}
\end{array}\right] \text {. } \\
& m \Pi^{\prime} \cdot\left[\begin{array}{c}
i_{12} \cdot\left(\ddot{q}_{4}+\ddot{q}_{5}\right) \\
\ddot{q}_{4}+\ddot{q}_{5} \\
\ddot{q}_{4}+\ddot{q}_{5} \\
0
\end{array}\right]=m \cdot\left(\Pi^{\prime}\right)^{2} \cdot i_{12} \cdot \mathbf{I h} \cdot \ddot{\mathbf{q}}+m \cdot \Pi^{\prime} \cdot \mathbf{I m} \cdot \ddot{\mathbf{q}}+m \cdot \Pi^{\prime \prime} \cdot i_{12} \cdot \mathbf{I h} \cdot \dot{\mathbf{q}}
\end{aligned}
$$




$$
\text { where: } \quad \mathbf{h}=\left[\begin{array}{llll}
i_{12} & 1 & 1 & 0 \\
i_{12} & 1 & 1 & 0 \\
i_{12} & 1 & 1 & 0 \\
0 & 0 & 0 & 0
\end{array}\right] \quad, \quad \mathbf{m}=\left[\begin{array}{cccc}
0 & 0 & 0 & i_{12} \\
0 & 0 & 0 & 1 \\
0 & 0 & 0 & 1 \\
0 & 0 & 0 & 0
\end{array}\right] .
$$

Then the system of equations describing the mechanical part of the drive can be represented by a matrix equation of the form:

$$
\begin{aligned}
& \left(\mathbf{M n}+\mathbf{I h} \cdot m \cdot\left(\Pi^{\prime}\right)^{2} \cdot i_{12}+\mathbf{I m} \cdot m \cdot \Pi^{\prime}\right) \cdot \ddot{\mathbf{q}}+\left(\mathbf{H n}+\mathbf{I h} \cdot m \cdot \Pi^{\prime} \cdot \Pi^{\prime \prime} \cdot i_{12}\right) \cdot \dot{\mathbf{q}}+\mathbf{C q}=\mathbf{Q m}, \\
& \mathbf{Q m}=\left[\begin{array}{c}
M d-\Pi^{\prime} \cdot i_{12} \cdot(m g+F r e z) \\
-\Pi^{\prime} \cdot(m g+F r e z) \\
-\Pi^{\prime} \cdot(m g+F r e z) \\
-m g-F r e z
\end{array}\right]=\left[\begin{array}{ccc}
1 & -\Pi^{\prime} \cdot i_{12} & -\Pi^{\prime} \cdot i_{12} \\
0 & -\Pi^{\prime} & -\Pi^{\prime} \\
0 & -\Pi^{\prime} & -\Pi^{\prime} \\
0 & -1 & -1
\end{array}\right] \cdot\left[\begin{array}{c}
M d \\
m g \\
F r e z
\end{array}\right], \\
& \mathbf{M} \cdot \ddot{\mathbf{q}}+\mathbf{H} \cdot \dot{\mathbf{q}}+\mathbf{C q}=\left[\begin{array}{ccc}
1 & -\Pi^{\prime} \cdot i_{12} & -\Pi^{\prime} \cdot i_{12} \\
0 & -\Pi^{\prime} & -\Pi^{\prime} \\
0 & -\Pi^{\prime} & -\Pi^{\prime} \\
0 & -1 & -1
\end{array}\right] \cdot\left[\begin{array}{c}
M d \\
m g \\
F r e z
\end{array}\right]=\mathbf{P v} \cdot\left[\begin{array}{c}
M d \\
m g \\
F r e z
\end{array}\right], \\
& \mathbf{M} \cdot \ddot{\mathbf{q}}+\mathbf{H} \cdot \dot{\mathbf{q}}+\mathbf{C q}=\left[\begin{array}{cc}
1 & -\Pi^{\prime} \cdot i_{12} \\
0 & -\Pi^{\prime} \\
0 & -\Pi^{\prime} \\
0 & -1
\end{array}\right] \cdot\left[\begin{array}{c}
M d \\
m g+F r e z
\end{array}\right]
\end{aligned}
$$

Thus, we have obtained a second-order matrix nonlinear differential equation for selected generalized coordinates. For further research, it is desirable to have a system of differential equations of the first order. To this end, we perform the following transformations: we consider matrices as components of block matrices:

$$
\begin{array}{cc}
\mathbf{R}=\left[\begin{array}{cc}
\mathbf{N u l M} & \mathbf{M} \\
\mathbf{M} & \mathbf{H}
\end{array}\right], \quad \mathbf{K}=\left[\begin{array}{cc}
-\mathbf{M} & \mathbf{N u I M} \\
\mathbf{N u l M} & \mathbf{C}
\end{array}\right], \quad \mathbf{Q}=\left[\begin{array}{c}
\mathbf{N}(4 \times 2) \\
\mathbf{P v}
\end{array}\right], \\
\mathbf{X}=\left[\begin{array}{c}
\dot{\mathbf{q}} \\
\mathbf{q}
\end{array}\right] .
\end{array}
$$

The system of differential equations of the first order can be written in the form:

$$
\mathbf{R} \cdot \dot{\mathbf{X}}+\mathbf{K} \cdot \mathbf{X}=\mathbf{Q} \cdot \mathbf{u}
$$


Where:

$$
\mathbf{u}=\left[\begin{array}{c}
M d \\
m g+F r e z
\end{array}\right]
$$

We check the transformations made:

$$
\left[\begin{array}{cc}
\mathbf{N}(4 \times 4) & \mathbf{M} \\
\mathbf{M} & \mathbf{H}
\end{array}\right] \cdot\left[\begin{array}{c}
\ddot{\mathbf{q}} \\
\dot{\mathbf{q}}
\end{array}\right]+\left[\begin{array}{cc}
-\mathbf{M} & \mathbf{N}(4 \times 4) \\
\mathbf{N}(4 \times 4) & \mathbf{C}
\end{array}\right] \cdot\left[\begin{array}{c}
\dot{\mathbf{q}} \\
\mathbf{q}
\end{array}\right]=\left[\begin{array}{c}
\mathbf{N}(4 \times 2) \\
\mathbf{P v}
\end{array}\right] \cdot \mathbf{u},
$$

The result is:

$$
\begin{gathered}
\ddot{\mathbf{q}} \cdot \mathbf{M}+\mathbf{H} \cdot \dot{\mathbf{q}}+\mathbf{C} \cdot \mathbf{q}=\mathbf{P v} \cdot \mathbf{u}, \\
\mathbf{M} \cdot \dot{\mathbf{q}}-\mathbf{M} \cdot \dot{\mathbf{q}} \equiv \mathbf{N}(4 \times 1)
\end{gathered}
$$

This result means that we have obtained a system of equations describing the behavior of the mechanism under study in state variables.

$$
\begin{gathered}
\Pi^{\prime}(\varphi)=-L \cdot \frac{d \delta}{d \varphi} \sin \delta+R \cdot \cos \varphi=L \cdot \varsigma \cdot \frac{\sin \varphi}{\cos \delta} \cdot \sin \delta+R \cdot \cos \varphi \\
\Pi^{\prime \prime}(\varphi)=L \cdot \frac{\sin \delta}{\cos \delta} \cdot\left(\varsigma^{2} \cdot\left(\frac{\sin \varphi}{\cos \delta}\right)^{2}-\varsigma \cdot \cos \varphi\right)-L \cos \delta \cdot\left(\varsigma \cdot \frac{\sin \varphi}{\cos \delta}\right)^{2}-R \cdot \cos \varphi
\end{gathered}
$$

\section{Conclusion}

The resulting system of drive equations can then be used to analyze the ratio of the spectrum of vibrations excited by the drive, both due to its dynamic properties, and due to the substantial nonlinearity of the law of motion of the working body and natural frequencies. In addition, it can be used in the analysis of forced movements.

\section{References}

1. V. Zhuravlev, V. Bespalov, Proceedings of universities. Construction and architecture, 10, 78-82 (1988)

2. B. Meskhi, A. Lee, V. Tsvetkov, Sat scientific Tr., 1, 3-12, GOU DPO "IUI AP", Rostov-on-Don (2004)

3. A. Lee, Sat. tr. scientific tech. Conf., 199-202, Rostov-on-Don (2005)

4. A. Chukarin, Theory and methods of acoustic calculations and design of technological machines for machining, 152, DGTU, Rostov-on-Don (2005)

5. A. Litvinov, A. Avakyan, I. Morozkin, Vestnik DGTU, 6, 57, 897-900 (2011)

6. D. Kozyrev, A. Avakyan, Bulletin of the DGTU, 2, 63, 1, 33-41(2012) 
7. A. Avakyan, V. Finochenko, Bulletin of the DGTU, 2, 63, 2, 5-11 (2012)

8. A. Avakyan, M. Scherba, Sat. Art. international scientific practical conf. "Metmash. Stankoinstrument" in the framework of the VII ind. Kongr Southern Russia, 346-348, Rostov-on-Don (2011)

9. A. Avakyan, Sat. Art. International Scientific Pract. Conf. "Metmash. Stankoinstrument" in the framework of the VII ind. Kongr Southern Russia, 346-348, Rostov-on-Don (2019) 